\title{
PRACTICAL NEUROLOGY
}

\section{Chronic Paediatric Neurological Disorders-Part I}

\author{
J. WILSON,* PH.D., M.R.C.P.
}

British Medical fournal, 1969, 4, 152-154

$$
\text { ". . . to comfort always." }
$$

The neurological disorders of childhood (with which mental subnormality should also be included) are neither infrequent nor are they trivial in terms of their impact on the lives of the patients themselves, on their families, and on the community. While prevention of these conditions must be the reasonable goal of medical science, it is unlikely that there will be a substantial reduction in their numbers for some time, and an increase in their frequency might even be expected if successful efforts to reduce infant and neonatal mortality do not also produce a favourable impact on morbidity rates.

\section{Community Care}

As community care is elaborated and the number of potential advisers multiplies, doctors, social workers, speech therapists, physiotherapists, teachers, and voluntary agencies may represent a potential wealth of help but also a bewildering array of conflicting and confusing advice. It may well be the task of the directors of the proposed district assessment centres to co-ordinate a structured programme of help for handicapped children, but it is nevertheless open to the parents to choose to whom they will impart their confidence. The family doctor, readily able to encompass the whole family in his interest and probably knowing the community much more intimately than either a hospital or town-hall-based doctor (both of whom may be forced to practise a littoral style of family medicine) may prove better able to understand his patient's needs than either of his more specialized counterparts, as well as being perhaps the first source of help to be approached. His initiative and co-ordination between the various agencies concerned with a neurologically handicapped child's welfare may help to resolve some of the social as well as medical difficulties described later which specialists recognize but are unable to avert. Unfortunately two important factors are likely to limit a family doctor's success in this sphere: firstly, lack of time, and, secondly, communication difficulties inhibiting the essential exchange of information.

\section{Co-ordination of Services}

Because of the chronic nature of many childhood neurological problems, and because a lot of effort may be rewarded by little obvious benefit, at least in the short-term, a practitioner may feel that the time-consuming repetition of complaints and difficulties conflicts too acutely with his other commitments. Moreover, though the best results come from direct discussion, this ideal can rarely be achieved. Exchange of correspondence, even if undertaken faithfully, is only second best, and does not provide a forum for the discussion which is essential for maraging complex conditions. As a result, help may be frustratingly fragmented and evoke antagonism rather than participation in the family " helped," and hostility between the helpers.

* Consultant Neurologist, Hospital for Sick Children, London.
These difficulties are mostly inherent in the nature of the patient's condition rather than in the perversity of the participants.

An extreme example is provided by Sally, who was referred to me by her family doctor for a second opinion at the age of 5 years. Some weeks earlier she had developed a hemiparesis accompanied by focal fits, an uncommon but well-recognized condition. She had been skilfully and fully investigated as an inpatient at a district hospital by a highly competent paediatrician, but the hostile relationship which developed between her "difficult" parents and hospital staff led to mutual disenchantment, and the disgruntled parents demanded referral elsewhere. Although when first seen at the Hospital for Sick Children superficial bruising possibly indicated a haemorrhagic tendency (a gross intracerebral or subdural collection having been excluded) it was later clear that she was subjected to a good deal of physical hurt at the hands of her stepfather, an irascible, illiterate psychopath who had been the main abrasive during the earlier hospital admission, and who had a seemingly immutable belief that the child was "lazy" down her affected side. The child's mother, a frail and partly deaf young woman, was estranged from her family in Ireland and found life in London lonely and threatening. The family, comprising two sisters and an infant step-brother, lived in a squalid and insanitary upstairs flat. The child's physical and mental condition deteriorated, and her behaviour regressed. The local authority provided sympathetic assistance over visits to hospital, help with other members of the family, schooling, and housing, and several months elapsed.

By now, the following medical and social agencies were involved: family doctor, district hospital and associated paediatric staff, neurological unit at the Hospital for Sick Children, with its associated staff and medical social worker; local authority staff-principal school medical officer, divisional educational officer, various assistant medical officers, mental welfare officer, housing officer, and health visitors.

The family was rehoused in another borough, where a different health visitor and an unfamiliar social worker and medical officers were introduced. The new accommodation was soon verminous, and there were acrimonious exchanges between health visitor and parents about the source of the lice. When, in one short period, the family was visited successively by a health visitor, a school attendance officer, an assistant medical officer, and a community social worker, all bent on helping, they refused to allow any more "interference," and subsequently maintained regular contact only with their family doctor. Though he was also relatively new, he conformed more closely to the parents' idea of a general practitioner's proper role-that of a prescriber of drugs and advice-and they were ready to accept his help. Unfortunately, even his reputation became rapidly tarnished when a prescribing mistake resulted in his patient developing phenytoin intoxication.

This extreme example is selected not so much to underline the complexity of the medical and social problems of one family (which is not uncharacteristic) as to emphasize that, on occasions, the medley of help involved may be self-defeating, and one person-for example, the family doctor-may need to feel personally concerned and personally involved.

\section{The Approach}

Even trivial illness in a child may arouse great anxiety in a family, and usually it is possible either to allay groundless fears 
or to provide support in a crisis. However, when that illness not only affects the brain but brings with it the prospect of permanently handicapping sequelae, it presents an array of family problems and attitudes which differ both in quality and in intensity from the reactions to other chronic maladies, and it is with these problems that this article is especially concerned.

In discussing the management of neurological disease in childhood one must not only separately consider acute and chronic illness but also acknowledge the special difficulties arising because the patient is a child whose health and whose future independence are the focus of hopeful anticipation by both parents and community. Only chronic neurological problems will be considered here, and the approach to the management of neurologically handicapping conditions in general will be illustrated by discussing separately three relatively common groups of disorders: cerebral palsy, epilepsy, and progressive degenerative disease. The management of mental subnormality is not considered, because though it is properly regarded as a neurological condition it deserves separate discussion along with learning difficulties generally.

\section{Cerebral Palsy}

In the United Kingdom there is a minimum prevalence of cerebral palsy of approximately 4 per 1,000 children of school age. This means that most family doctors will have several children with cerebral palsy of varying degrees of severity in their practice.

Though the definition" of cerebral palsy as "a persistent but not unchanging disorder of movement and posture, appearing in the early years of life and due to a non-progressive disorder of the brain, the result of interference during its development" emphasizes the motor involvement, in the majority of patients there are multiple potentially handicapping disorders. The existence of sensory disturbance, visual abnormalities (optic atrophy, squint, nystagmus, hemianopia), hearing loss, mental subnormality, or specific learning difficulties must be identified if the child is to receive appropriate help. The need to make a careful appraisal of these multiple problems as a prerequisite for properly organized help has initiated moves to establish district assessment centres.

It is generally accepted that physical treatment may prevent serious deformities and provide general stimulation to a child whose inability to explore his environment may add sensory and social deprivation to his physical problems, but there is much debate about whether or not very early treatment during infancy can favourably influence the pattern of motor development and encourage more normal posture and use of affected limbs. If it could be demonstrated that regular treatment initiated during the first 12 months of life was convincingly superior to the present policy of attempting to deal with the abnormalities of tone and posture only when they are well enough established to be blatantly clear even to a casual observer, then early case-finding and pre-school treatment centres would demand a higher degree of priority than they do now. At present, with some notable exceptions, there is not much enthusiasm for the physical treatment of infants and the very young. This is regrettable-if only because of the impotent unease it encourages in mothers who may know that "something is wrong."

On the other hand, it is important to acknowledge that there is as yet no known cure for cerebral palsy. Parents may too readily be " hooked" on physiotherapy and conclude (like some therapists) that the more frequently treatment is given the more likely will their efforts be rewarded by "success." "Success" in this situation is relative, and if it is believed that normal development is a criterion of success, ensuing disappointment at its "failure" may be accompanied by a sense of profound guilt that the treatment has not been carried out properly. This is perhaps the most repugnant aspect of one system of treatment which claims return to normality (or even "super-normality") as its usual goal, and implies that any shortcomings are the fault of the parents applying the scheme, and not the product of its own intemperate fantasy.

\section{Diagnosis}

At present in Britain the diagnosis is usually established in pre-school years, though a certain number of children with, for example, mild hemiplegic cerebral palsy may be referred to an orthopaedic clinic with postural abnormalities of hand or foot at later ages.

Though a label of " cerebral palsy" still tends to be loosely applied to any neurodevelopmental disorder of early life, it is usually possible to establish the diagnosis more strictly. A doctor with a general approach should be allowed to review the needs of the child, because of the frequent association with other neurological deficits, all of which need to be systematically assessed, and for which a properly integrated programme of help needs to be organized.

No matter how young the child, it is usually possible to make at least tentative assessments of some of these problems, and then to make arrangements for inclusion of the child into a pre-school programme of treatment. For the very young, this may be no more than passive stimulation and movement and an awakening of interest in under-used limbs, but in toddlers inclusion in a nursery group allows the beginning of the social integration which will assume increasing importance as the child gets older, and also provide an impetus to activity and better co-ordination, together with an increasing sense of independence through play, and education in its widest sense.

Before starting school, important decisions concerning educability and the need for special education at schools for the physically handicapped can also be based on a period of regular observation in a learning situation and on serial psychological examinations.

Mothers (and some fathers) benefit from contact with a preschool treatment group orientated to their needs, as well as those of their children, where they can also meet parents of other handicapped children. Mothers of young handicapped children who attend ordinary physiotherapy units at general hospitals sometimes complain that they sense an indifference to their particular problems, and often feel that no other parent has a similarly affected child.

Though the provision of appropriate schooling is statutorily defined, and in recent years there has been a steady improvement in facilities, it is not always possible for a child to be suitably placed at the age of 5. Moreover, some parents are very reluctant to accept the need for other than "normal" schooling, and in children whose handicap is not severe and whose intelligence is borderline it is the policy of some local authorities to allow a period at a normal school.

The clinical evolution of cerebral palsy is most conspicuous during the early years, but continues to some extent throughout the period of growth and adolescence. This is not due to change in the underlying organic cerebral deficit so much as to the interaction of physical growth and abnormal posture, though maturational changes in the brain probably do occur. Regular review is therefore necessary in case orthopaedic manœuvres are necessary.

\section{Adolescence}

The social and psychological aspects of cerebral palsy will be discussed later. But the problems of the adolescent with cerebral palsy are sufficiently important to merit separate mention. Adolescence can be a time of great anguish for the patient and his family. Self-consciousness about physical difficulties at a time of heightening sexual awareness may bring a depressing 
sense of inadequacy and frustration. The problems of achieving economic independence may provide a strident reminder that elaborate and assiduous efforts of earlier years may be utterly frustrated if high national unemployment rates threaten the work prospects of a young man with a moderate physical handicap.

\section{Epilepsy}

The management of recurrent seizures in childhood is coloured by similar psychosocial problems to those besetting children with cerebral palsy and their families. There is some evidence to suggest that they are indeed more common. ${ }^{2}$ The complicating side-effects of drugs, the prejudicial fearfulness of many lay people, including teachers, the tendency to parental over-protection, and not least the peculiarity of some epileptic attacks themselves combine to provide a stress which for some families is well-nigh intolerable.

On the other hand, certain seizure disorders in childhood are so benign that optimism is nearly always justified. So-called "febrile convulsions" tend to occur between the ages of 1 and 5 and may represent a genetically determined tendency to convulse during an otherwise trivial infective illness, not necessarily at a stage when fever is evident. Though they are usually considered to be generically and prognostically distinct from other seizure disorders, the work of Ounsted and his colleagues ${ }^{3}$ provides important evidence that febrile convulsions may themselves have a potentially permanent damaging and self-potentiating effect, and thus to be an important aetiological factor in more chronic forms of epilepsy. If Ounsted's studies are validated, then readiness to dismiss "febrile convulsions" as invariably inconsequential must be abandoned, and steps taken to ensure that the duration of any fits, whatever the cause, is minimized. The drug treatment of epilepsy in childhood will be discussed in a later article, but drugs are not the whole answer to the problem. Psychological factors often determine the frequency of fits, and their recognition can often provide the key to a greater degree of therapeutic success eithor by a family doctor or, in the more difficult cases, by a psychiatrist.

Sympathetic help enables many epileptic children to participate fully in the life of an ordinary school, but, depending on the fit frequency, the degree of acceptance and understanding by the teaching staff, and the child's educational ability, altornative placement may have to be sought. Since fits are symptomatic of conditions which may also manifest themselves by physical handicap and by mental subnormality, a considerable number of children with fits attend schools for the educationally subnormal (E.S.N.), for the physically handicapped (P.H.), or junior training centres. There are additionally a small number of residential schools specifically for epileptic children.

The question of whether or not to allow epileptic children to swim, climb, or ride a bicycle is a difficult one to answer. The need to avoid giving a frustratingly restricted view of future activities has to be balanced against the real hazard to the child, to other road users, and the anxiety of teachers in loco parentis. It is most unlikely that a reasonably controlled epileptic child will have a fit while engaged in such enjoyable activities as cycling and swimming. It is difficult to define what is meant by "reasonably controlled," but in general I consider a child reasonably controlled if the frequency of seizures has been reduced to one per fortnight or less, if fits occur at reasonably well-defined times-nocturnal or first thing in the morning-and are preferably accompanied by some prodromal warning. Each case must be considered on its merits, and I usually prefer to postpone a decision for six months after starting treatment until its effect has been judged. If allowed, swimming should be supervised.

(The conclusion of this article, with a list of references, will be printed next week.)

\section{ANY QUESTIONS?}

We publish below a selection of questions and answers of general interest.

Tea, Coffee, and Migraine

Q.-Are tea and coffee known to cause attacks of migraine in some patients, and, if so, why?

A.-In my experience neither tea nor coffee acts as a provoking factor in cases of migraine. Indeed, caffeine, either alone ormore often-in association with ergotamine, is commonly found to be of some benefit in relieving an actual attack.

\section{Hypersensitivity to Milk}

Q.-Is a diagnosis of anaphylaxis due to hypersensitivity to milk scientifically defensible?

A.-Milk allergy in infants may present with severe gastrointestinal symptomsvomiting, diarrhoea, and rectal bleeding. Biopsy of the rectal mucosa may show large numbers of eosinophils in the lamina propria, and there is likely to be an increase in the eosinophil count in the circulating blood. Serological investigations show a titre of "whole-milk antibody" many times above the accepted normal level of $1 / 20$ $1 / 40 .^{1}$ The diagnosis is confirmed by the subsidence of symptoms after removal of milk from the diet and their reappearance within 48 hours of trial feeding of milk repeated on three successive occasions.

Using such strict criteria one institution collected some 21 cases in 16 years. ${ }^{2}$ Seven of the patients showed signs of cardiovascular collapse when challenged with milk. True milk allergy is thus relatively rare, and only a small proportion of affected infants appear to suffer from shock when milk is exhibited in the diet. Infants who die unexpectedly do not as a rule have a high titre of circulating antibodies to cow's milk.

\section{REFERENCES}

1 Silver, H., and Douglas D. M., Archives of

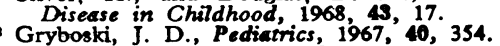
2 Gryboski, J. D., Pediatrics, 1967,

\section{Tonsillectomy and Virus Infections}

Q.-Is a person more prone to poliomyelitis and other virus infections, such as glandular fever, after tonsillectomy?

A. - There is no evidence that tonsillectomy increases susceptibility to poliomyelitis or other virus infections such as glandular fever. However, people who have undergone tonsillectomy and who subsequently develop poliomyelitis are more prone to develop the bulbar form of the disease. The risk of bulbar involvement is highest in those who have had a tonsillectomy 7 to 21 days before the onset of poliomyelitis, ${ }^{1}$ but there is an increased risk even in those who have had their tonsils removed many years previously.

\section{RBPERENCES}

1 Aycock, W. L., Medicine, 1942, 21, 65

Anderson. G. W. ., and Rondeau, J. L., fournal of the American Medical Association, 1954, 155,1123 .

\section{Notes and Comments}

Topical Insulin.-Dr. A. SAxula (Redhill General Hospital, Redhill, Surrey) writes: With reference to the answer to this question ("Any Questions?" 20 September, p. 703) your expert stated that he had been unable to find any reference to this subject. May I draw his attention to the account by Paul $^{1}$ of a diabetic patient of mine in whom insulin applied locally to the infested wound of an amputation stump led to a dramatic cure when previous treatment by antibiotics alone had failed to achieve this ? Subsequent correspondence ${ }^{2}$ s revealed that this phenomenon had been observed by others. I appreciate that my case was not strictly that of a penetrating ulcer, but on the analogy I consider that there is evidence that locally applied insulin is worth trying in these cases.

\section{REFERENCES}

1 Paul, T. N., Lancet, 1966, 2, 574 Paul, T. N., Lancet, 1966, 2, 574.
Csapó, G., and Hódi, M., Lancet, 1966, 2, 1466.
Naiji, K., Lancet, 1967, 1, 394. 